\title{
Síndrome febril prolongado de origen reumatológico. Reporte de tres casos
}

\author{
Renato Antonio Guzmán ${ }^{1}$, Maria Camila Mejía ${ }^{2}$, Vanessa Cadavid ${ }^{3}$, María Claudia Barrera ${ }^{3}$.
}

1. Médico Internista, Inmunólogo-Reumatólogo IDEARG.

2. Médico estudiante de Epidemiología - Fundación Universitaria Juan N. Corpas.

3. Médico general Servicio Social Obligatorio - Centro de Investigación, Fundación Universitaria Juan N. Corpas.

Autor de correspondencia:

renato.guzman@juanncorpas.edu.co

Como citar: Guzmán RA, Mejía MC², Cadavid V, Barrera MC. Síndrome febril prolongado de origen reumatológico. Reporte de tres casos. Revista Cuarzo 2018: 24 (1) 46-49.

Recibido: 17 de mayo de 2018 Aceptado: 25 de junio de 2018 Publicado: 30 de junio de 2018

Licencia creative commons (c) $($ ) $\circledast \Theta$ BY NC NO

\section{Resumen}

El presente trabajo describe tres casos con Síndrome Febril Prolongado (SFP) con diagnóstico definitivo de enfermedad reumatológica, el primero, hombre de 29 años con picos febriles de $40^{\circ} \mathrm{C}$, mialgias y adenopatías cervicales; único antecedente, viaje 30 días previos a región tropical, lo que hizo pensar en etiología infecciosa, sin embargo, tras persistencia de fiebre a pesar de cubrimiento antimicrobiano, se extienden estudios paraclínicos con hallazgo de ferritina en $2208 \mu \mathrm{g} / 1$ sugestivo de Enfermedad de Still del adulto al décimo día de estancia. El segundo es un hombre de 19 años, con clínica de dolor abdominal asociado a temperatura de $40^{\circ} \mathrm{C}$ sin antecedentes de importancia; ecografía abdominal evidencia hepatomegalia y derrame pleural; durante la estancia, presentó convulsiones tónico-clónicas y pancitopenia con anemia hemolítica, posterior a descartar cuadro infeccioso y neoplasia maligna, se solicitó perfil inmunológico con ANAS y Anticoagulante Lúpico positivos y se orienta diagnóstico hacia Lupus Eritematoso Sistémico al onceavo día de hospitalización, con mejoría posterior al manejo inmunomodulador. Por último, paciente femenina de 14 años con historia de artralgias, adenopatías cervicales/submaxilares, exantema morbiliforme y fiebres fluctuantes $\left(38-39,9^{\circ} \mathrm{C}\right)$ de un mes de evolución que persistía pese a policultivos negativos y antibioticoterapia de amplio espectro por lo cual se amplían estudios de laboratorio encontrando ferritina de $3156 \mu \mathrm{g} / \mathrm{l}$ al día noveno de estancia y se diagnostica Enfermedad de Still del niño. La prevalencia de enfermedades reumatológicas en el SFP, ha venido en aumento permitiendo la aproximación precisa a otras etiologías antes no consideradas entre sus causas.

Palabras clave: síndrome febril; enfermedad autoinmune; reumatología.

\section{Prolonged febril syndrome of rheumatological origin. Report of three cases}

\begin{abstract}
The present work describes three cases with Prolonged Febrile Syndrome (PFS) with definitive diagnosis of rheumatological disease. The first, a 29 -year-old man with febrile peaks of $40{ }^{\circ} \mathrm{C}$, myalgias and cervical adenopathies; trip 30 days ago to tropical region, which led to think about an infectious etiology; however, after persistence of fever despite antimicrobial coverage, paraclinical studies are extended with ferritin finding at $2208 \mu \mathrm{g} / 1$ suggestive of Still's Disease from the adult to the tenth day of stay. The second is a 19-year-old man, with symptoms of abdominal pain associated with a temperature of $40^{\circ} \mathrm{C}$ with no relevant history; Abdominal ultrasound evidences hepatomegaly and pleural effusion; during the stay, presented tonic-clonic seizures and pancytopenia with hemolytic anemia, after ruling out infectious disease and malignancy, an immunological profile with positive ANAS and Lupus Anticoagulant was requested and a diagnosis was made towards Systemic Lupus Erythematosus on the eleventh day of hospitalization, with improvement after immunomodulatory management. Finally, a female patient of 14 years with a history of arthralgias, cervical and submaxillary adenopathies, morbilliform exanthema and fluctuating fevers (38-39.9 ${ }^{\circ}$ C) of a month of evolution that persisted despite negative polycultures and broad spectrum antibiotic therapy. which are extended laboratory studies finding ferritin of $3156 \mu \mathrm{g} / 1$ on the ninth day of stay and is diagnosed with Still's disease of the child. The prevalence of rheumatological diseases in the PFS, has been increasing allowing the precise approach to other etiologies previously not considered among its causes.
\end{abstract}

Keywords: febrile syndrome; autoimmunity disease; rheumatology. 


\section{Introducción}

El síndrome febril prolongado (SFP) se define como una temperatura axilar mayor a $38,3^{\circ} \mathrm{C}$ tomada al menos dos veces en un periodo de tres semanas o después de 3 días de hospitalización o 3 consultas ambulatorias sin diagnóstico concluyente; puede explicarse desde diversas etiologías agrupadas en: enfermedades infecciosas (25-40\%), neoplasias (15-30\%), enfermedades inflamatorias no infecciosas (10-30\%), otras causas vasculares, metabólicas o farmacológicas (5-15\%) y aquellas de origen desconocido (FOD) que cesan sin un diagnóstico establecido (1-3). Actualmente, la FOD es menos frecuente, contribuyendo al aumento en los otros grupos de clasificación etiológica, particularmente al de enfermedades inflamatorias no infecciosas ya que el avance en el diagnóstico serológico ha permitido la aproximación más precisa y rápida a las enfermedades del tejido conectivo y entre estas las más frecuentemente relacionadas con FOD son: enfermedad de Still, polimialgia reumática, arteritis temporal, artritis reumatoide, fiebre reumática, enfermedad inflamatoria intestinal, síndrome de Reiter, lupus eritematoso sistémico y vasculitis $(3,4)$.

A pesar de lo descrito, el paciente con SFP, adulto o pediátrico, continúa siendo un reto diagnóstico para el médico en las diferentes áreas, sea por la heterogeneidad clínica y paraclínica a través del tiempo o por la necesidad de instaurar un tratamiento efectivo $(5,6)$. A continuación se presentan tres casos clínicos con SFP como manifestación de enfermedades reumatológicas en diferentes grupos etarios.

\section{Presentación de casos}

\section{Caso 1}

Paciente masculino de 29 años de edad natural de Caracas, residente en Bogotá, ocupación odontólogo, consultó por cuadro clínico de 8 días de evolución consistente en fiebre cuantificada en $40^{\circ} \mathrm{C}$ que relaciona con abrasión cutánea en cara anterior de miembro inferior izquierdo hace 8 días y viaje a clima tropical hace 30 días; asocia mialgias generalizadas, astenia, adinamia y adenopatías cervicales e inguinales bilaterales, manejado ambulatoriamente con Dicloxacilina $500 \mathrm{mg}$ cada 12 horas, Acetaminofén $500 \mathrm{mg}$ cada 8 horas y Diclofenaco $50 \mathrm{mg}$ cada 8 horas sin mejoría de lo descrito, además manifiesta pérdida de $4 \mathrm{Kg}$ en la última semana. Refiere que es la primera vez que presenta la sintomatología.

Al indagar sobre sus antecedentes, refiere diarrea crónica intermitente desde hace 5 años, comenta ser consumidor ocasional de sustancias psicoactivas y fumador de 5 cigarrillos/día desde los 20 años hasta 3 meses previos al ingreso, traumatológicos, farmacológicos y epidemiológicos descritos en enfermedad actual. En el examen físico de ingreso los signos vitales evidenciaban taquicardia (>100 lpm) e hipertensión arterial (140/92 mmHg), como hallazgos positivos se palpan adenopatías retroauriculares y occipitales no dolorosas e inguinales bilaterales dolorosas, miembro inferior izquierdo con lesión no dolorosa tipo abrasión en región tibial anterior de $3 \mathrm{~cm}$ sin signos de inflamación o infección; resto del examen físico normal.

Se decide hospitalizar por el servicio de Medicina Interna con diagnóstico de FOD y síndrome constitucional, se inicia antibioticoterapia de amplio espectro con Vancomicina + Ceftriaxona, control de signos vitales y se solicitan paraclínicos para estudio entre estos, Tomografía Axial Computarizada (TAC) de tórax y abdomen. ecocardiograma, cuadro hemático, parcial de orina, urocultivo, hemocultivos, serología VDRL, VIH, Hepatitis B y C, Citomegalovirus, Toxoplasmosis, Leptospira, cultivo para Leptospira en orina, látex para mononucleosis infecciosa, pruebas de función hepática y PCR de los cuales solo se evidenció alteración en Alanino-aminotransferasa (ALT) en $103 \mathrm{U} / \mathrm{L}$ y en la PCR en $20 \mathrm{mg} / \mathrm{dl}$. Descartado todo lo anterior, hacia el cuarto día de estancia hospitalaria, se presenta pico febril de $38,5^{\circ} \mathrm{C}$ a pesar de manejo antibiótico, se considera el estudio de causas inflamatorias secundarias a procesos infecciosos, enfermedades del tejido conectivo y neoplasias con TAC de senos paranasales, cráneo y conducto auditivo, nuevos policultivos (hemocultivos, urocultivo, coprocultivo), coprológico, serología para Dengue, gota gruesa, perfil inmunológico habitual, Ferritina, PCR, TSH y se interconsulta por infectología para concepto, quienes indican inicio escalonar a Ampicilina/Sulbactam 3 gr cada 6 horas hasta tener reporte de cultivos.

$\mathrm{Al}$ sexto día desde el ingreso, se registra fiebre de $38,3^{\circ} \mathrm{C}$ asociado a dolor en plantas de pies y rodillas; por antecedente patológico de diarrea crónica, se solicita valoración por gastroenterología para pesquisa de tumor maligno gastrointestinal quienes solicitan estudios endoscópicos si no se encontrase etiología del cuadro. En el octavo día, se evidencia disminución en las adenopatías referidas al ingreso, sin embargo persiste dolor en plantas de pies y rodillas y se tiene reporte parcial de perfil inmunológico el cual evidencia ANAS 1/160 y Ferritina en 2208 $\mu \mathrm{g} / \mathrm{l}$ (positivos) a lo cual el servicio de Reumatología diagnostica Enfermedad de Still de adulto e inicia manejo con Prednisolona $20 \mathrm{mg}$ cada 24 horas, Metotrexate $10 \mathrm{mg}$ cada semana, Ácido fólico $1 \mathrm{mg}$ cada 24 horas. En los siguientes dos días, el paciente refiere mejoría de sintomatología presentada, sin fiebre, resuelve taquicardia e hipertensión, desaparecen adenopatías y se da egreso con orden para seguimiento ambulatorio por la especialidad.

\section{Caso 2}

Paciente masculino de 19 años de edad, natural y residente en Bogotá, empleado de call center, remitido de otra institución hospitalaria por cuadro clínico de 11 días de evolución consistente en fiebre cuantificada diariamente de $40^{\circ} \mathrm{C}$, asociado a astenia, adinamia, escalofríos, diaforesis nocturna, dolor abdominal tipo punzada en epigastrio, irradiado a región umbilical, de intensidad $8 / 10$, cuatro deposiciones líquidas fétidas, sin moco, ni sangre, un episodio de epistaxis y en el último día exantema facial. Se hospitalizó con diagnóstico de FOD y leucopenia, se inició manejo antipirético con Acetaminofén y se solicitaron 
paraclínicos de extensión: ecografía de abdomen evidenció derrame pleural laminar bilateral y líquido libre ascítico, tiempos de coagulación prolongados, cuadro hemático con pancitopenia, transaminasas, DHL, fosfatasa alcalina y TSH elevadas, coombs directo positivo con leve tinte ictérico. Dada la alteración de las pruebas hepáticas, se decide descartar enfermedad hepatocelular, absceso hepático. Entre el segundo y sexto día de hospitalización el paciente continuaba con deposiciones diarreicas, además, presentó un episodio emético y una convulsión tónico clónica que resuelve de manera espontánea, una nueva ecografía abdominal reportó hepatoesplenomegalia y derrame pleural bilateral confirmado por TAC-alta resolución (TACAR) con densidades alveolares mal definidas basales bilaterales por proceso parenquimatoso, atelectasia laminares y parahiliares; se inicia manejo con Piperacilina/Tazobactam y se indica toracentesis descompresiva.

Por episodio convulsivo referido, lo valora neurología quienes realizaron punción lumbar con reporte de estudios, negativos, sin embargo, considerando la presencia de compromiso neurológico, poliserositis, pancitopenia y ANAs positivos (1/320), se interroga Lupus Eritematosos Sistémico (LES) versus hepatitis autoinmune y se instaura manejo con metilprednisolona y se solicita valoración por el Servicio de Reumatología quien considera que el cuadro clínico orienta hacia LES, se adicionó Azatriopina y Cloroquina. Se evidenció mejoría de la sintomatología, no se registraron nuevos picos febriles y posteriormente se dió salida con orden para seguimiento ambulatorio por consulta externa de Reumatología.

\section{Caso 3}

Paciente femenina de 14 años de edad, natural y procedente de Bogotá, ocupación estudiante, remitida de otra institución hospitalaria en compañía de sus padres con cuadro clínico de aproximadamente un mes de evolución consistente en fiebre cuantificada en $38-39,9^{\circ} \mathrm{C}$, sin predominio horario, asociado a artralgias y mialgias generalizadas, por lo cual había consultado en dos ocasiones previas a su entidad de salud y fue manejada con diferentes esquemas antibióticos por diagnóstico de Faringoamigdalitis bacteriana, entre estos, Penicilina Benzatínica 1,200,000 UI dosis única y Amoxicilina $500 \mathrm{mg}$ cada 8 horas por 7 días, además, Acetaminofén $500 \mathrm{mg}$ cada 8 horas; sin embargo, al no evidenciar mejoría de los síntomas, los padres deciden consultar a urgencias de institución remisoria donde realizan paraclínicos: cuadro hemático con leucocitosis en aumento progresivo en 24 horas de 19,600 a $22,600 / \mathrm{mm} 3$ a expensas de neutrófilos, además elevación de reactantes de fase aguda PCR 96 mg/dl y VSG $39 \mathrm{~mm} / \mathrm{h}$, diagnostican fiebre de origen desconocido, inician cubrimiento con Ceftriaxona 1 gr cada 12 horas y remiten para estudios de extensión.

Familiares refieren que es la primera vez que presenta la sintomatología.

Según lo referido, no hay antecedentes patológicos, sin nexos epidemiológicos; farmacológicos descritos y familiares de importancia, madre tuvo Guillain Barré.
Al examen físico de ingreso, se encuentra taquicárdica (130 $\mathrm{lpm}$ ), leve eritema faríngeo, adenopatías submaxilares y cervicales dolorosas a la palpación en número de 3 de aproximadamente $0,5 \mathrm{~cm}$ de diámetro y exantema morbiliforme pruriginoso en abdomen y espalda.

Como conducta, se decide hospitalizar por el servicio de Pediatría para estudio del cuadro clínico con diagnóstico de síndrome febril de origen desconocido, inicialmente en búsqueda de foco infeccioso que lo explicara por lo que solicitan TAC de tórax, de senos paranasales y abdominal, ecografía abdominal, test rápido para Streptococcus betahemolítico y cultivos de sangre y orina, continúan manejo con antibiótico parenteral instaurado en centro de referencia y adicionan Hidroxicina Clorhidrato.

El reporte de estudios imagenológicos no describe alteraciones al igual que el Strepto-test. Hacia el tercer día de hospitalización, el exantema se extiende hacia miembros superiores e inferiores, continúa la artralgia y los picos febriles, sin aislamientos en los cultivos, por lo cual se sospecha enfermedad del tejido conectivo y se solicita perfil inmunológico, perfil tiroideo, ecocardiograma, electrocardiograma y radiografía de manos comparativa, en la que no se evidencian lesiones. En el cuarto día de su estancia en la clínica, persiste desviación a la izquierda de leucocitos, ahora en $24,200 / \mathrm{mm} 3$ aún a expensas de neutrófilos y como nuevo hallazgo anemia con hemoglobina de $11 \mathrm{gr} / \mathrm{dL}$. El reporte de perfil tiroideo es normal, ecocardiograma sin alteraciones y electrocardiograma con taquicardia sinusal, pendiente aún perfil inmunológico, fue valorada por servicio de Reumatología, quienes consideran solicitar Ferritina sérica por sospecha de Enfermedad de Still, se instaura tratamiento con AINE (Ibuprofeno $400 \mathrm{mg}$ cada 8 horas).

Un nuevo cuadro hemático evidencia mejoría de leve de leucocitosis con descenso glóbulos blancos absolutos a 20,200/mm3, sin embargo empeoramiento de la anemia con nuevo descenso de la hemoglobina en $9,9 \mathrm{gr} / \mathrm{dL}$, por lo cual, ante sospecha diagnóstica de enfermedad reumatológica, se solicita radiografía de tórax para descartar hemorragia alveolar, sin embargo, esta no se hace evidente y el perfil de hemólisis es negativo. En el día noveno, se tiene el reporte de perfil inmunológico el cual es negativo y de Ferritina positiva en $3156 \mu \mathrm{g} / \mathrm{l}$, por lo cual, reumatología define diagnóstico de Enfermedad de Still y trata con Prednisolona $5 \mathrm{mg}$ cada 12 horas, Metotrexate $10 \mathrm{mg}$ cada semana y Ácido fólico $1 \mathrm{mg}$ cada 24 horas; la paciente refiere mejoría de sintomatología respecto al ingreso y hacia el décimo día de estancia, egresó con orden médica para seguimiento ambulatorio por Reumatología pediátrica.

\section{Discusión}

El SFP se presenta con frecuencia en la práctica clínica, su abordaje se convierte en un desafío para llegar a un diagnóstico etiológico certero. La mayoría de los casos reportados en la literatura corresponden a causas infecciosas, entre las cuales se destacan rickettsiosis, babesiosis, fiebre tifoidea, mononucleosis infecciosa y en nuestro país, las más prevalentes han sido tuber- 
culosis, dengue y chicungunya (7-11); lo cual obliga a descartar inicialmente estas patologías, tal como se pensó en los tres casos descritos. En contraste con lo anterior, desde el 2007, en un estudio neozelandés posicionó las causas inflamatorias no infecciosas como la etiología predominante con el $22 \%$ de los 2800 pacientes incluidos, sin embargo, el 51\% de los pacientes no tuvo un diagnóstico definitivo (12), problema que viene en constante remisión con el progreso tecnológico en el ámbito de exámenes diagnósticos, permitiendo que las enfermedades del tejido conectivo sean un importante diagnóstico diferencial (3).

Según lo descrito en la literatura, la edad de los pacientes es determinante para establecer el diagnóstico, por ejemplo, en los pacientes pediátricos, la mayoría de las etiologías son infecciosas-virales y autoresuelven, sin embargo, es pertinente descartar neoplasias malignas, las cuales corresponden a un alto porcentaje en esta edad; así, en adultos, aunque también predominan las infecciosas, para ambos tipos de pacientes, en la última década se ha evidenciado aumento en aquellas de causa reumatológica, llegando casi a compararse con los grupos mencionados (1315).

Los tres pacientes de este reporte cumplieron con la definición del SFP presentando temperatura axilar mayor a $38,3^{\circ} \mathrm{C}$ dos veces después de 3 días de hospitalización. En los tres casos, considerando la prevalencia de enfermedades infecciosas como etiología del SFP, se realizaron los estudios necesarios para descartar Mononucleosis infecciosa, Hepatitis B, Hepatitis C, Citomegalovirus, Epstein Barr, VIH, Sífilis, Tuberculosis, Dengue, Leptospirosis y aquellas que para la edad del paciente pediátrico pudieran asociarse. Luego de descartarse el origen infeccioso del SFP, los exámenes del perfil inmunológico y los específicos como los niveles de Ferritina, en paralelo con los resultados imagenológicos y la clínica de los pacientes permitieron identificar los criterios diagnósticos de las dos enfermedades de tipo reumatológico que explicaban la fiebre prolongada de los pacientes.

\section{Conclusión}

El SFP es un diagnóstico frecuente en los servicios de urgencias y hospitalización, el cual se realiza previo al estudio de cualquier cuadro clínico que presente fiebre. Cada día podemos ver con mayor frecuencia que este síndrome se asocia a causas reumatológicas, por esto se hace importante hacer un llamado al personal de salud para estar alerta a la complejidad de los signos y síntomas que pueda presentar un paciente con características febriles y reumatológicas para dar un tratamiento efectivo.

\section{Referencias}

1. Durack DT, Street AC. Fever of unknown origin — reexamined and redefined. Curr Clin Top Infect Dis 1991;11:37.

2. Zenone T. Fever of unknown origin in adults: evaluation of 144 cases in a non-university hospital. Scand J Infect Dis. 2006;38(8):632-8.

3. Hayakawa K, Ramasamy B, Chandrasekar PH. Fever of unknown origin: an evidence-based review. Am J Med Sci. 2012;344(4):307-16.

4. Roth AR, Basello GM. Approach to the adult patient with fever of unknown origin. Am Fam Physician. 2003;68(11):2223-8.

5. Janow G, Schanberg LE, Setoguchi S, Hasselblad V, Mellins ED, Schneider R, et al. The Systemic Juvenile Idiopathic Arthritis Cohort of the Childhood Arthritis and Rheumatology Research Alliance Registry: 2010-2013. J Rheumatol. 2016;43(9):1755-62.

6. Balci MA, Pamuk ÖN, Pamuk GE, Uzundere FK, Donmez S. Epidemiology and outcome of adult-onset Still's disease in Northwestern Thrace region in Turkey. Clin Exp Rheumatol. 2015;33(6):818-23.

7. Sanhueza NC, Farías S, Calzadilla J, Hermoso A. Typhoid fever: case report and literature review. Medwave. 2016;16(5):e6474.

8. Lemieux JE, Tran AD, Freimark L, Schaffner SF, Goethert H, Andersen $\mathrm{KG}$, et al. A global map of genetic diversity in Babesia microti reveals strong population structure and identifies variants associated with clinical relapse. Nat Microbiol. 2016;1(7):16079.

9. Faccini ÁA, Botero CA, Hidalgo M. Contributions to rickettsioses research in colombia (1917-1943), luis b. Patiño camargo. Rev Inst Med Trop Sao Paulo. 2016;58:33.

10. Restrepo BN, Piedrahita LD, Agudelo IY, Marín K, Ramírez R. Dengue infection: A common cause of febrile syndrome in patients from Quibdó, Chocó, Colombia. Biomedica. 2015 Jan-Mar;35(1):131-7.
11. Abdelbaky MS, Mansour HE, Ibrahim SI, Hassan IA. Prevalence of connective tissue diseases in egyptian patients presenting with Fever of unknown origin. Clin Med Insights Arthritis Musculoskelet Disord. 2011;4:3341.

12. Bleeker-Rovers CP, Vos FJ, de Kleijn EM, Mudde AH, Dofferhoff TS, Richter $\mathrm{C}$, et al. A prospective multicenter study on fever of unknown origin: the yield of a structured diagnostic protocol. Medicine (Baltimore) 2007;86(1):26-38.

13. Bosilkovski M, Dimzova M, Stevanović M, Cvetkovska VS, Duganovska MV. Fever of unknown origin--diagnostic methods in a European developing country. Vojnosanit Pregl. 2016;73(6):553-8.

14. Turkulov V, Brkić S, Sević S, Marić D, Tomić S. Fever of unknown origin in elderly patients. Srp Arh Celok Lek. 2011;139(1-2):64-8.

15. Chien YL, Huang FL, Huang CM, Chen PY. Clinical approach to fever of unknown origin in children. J Microbiol Immunol Infect. 2015;S16841182(15)00830-0. 